\title{
Intelligent Cloud Learning Model for Online Overseas Chinese Education
}

\author{
http://dx.doi.org/10.3991/ijet.v10i1.4284 \\ Zhehuang Huang ${ }^{1,2}$,Xiaodong Shi ${ }^{*}$,Yidong Chen ${ }^{1}$ \\ ${ }^{1}$ Xiamen University, Xiamen, China \\ ${ }^{2}$ Huaqiao University, Quanzhou, China
}

\begin{abstract}
With the development of Chinese economy, oversea Chinese education has been paid more and more attention. However, the overseas Chinese education resource is relatively lack because of historical reasons, which hindered further development. How to better share the Chinese education resources and provide intelligent personalized information service for overseas student is a key problem to be solved. In recent years, the rise of cloud computing provides us an opportunity to realize intelligent learning mode. Cloud computing offers some advantages by allowing users to use infrastructure, platforms and software. In this paper we proposed an intelligent cloud learning model based on cloud computing. The learning model can utilize network resources sufficiently to implement resource sharing according to the personal needs of students, and provide a good practicability for online overseas Chinese education.
\end{abstract}

Index Terms-overseas Chinese education, cloud computing, online learning, student modeling.

\section{INTRODUCTION}

Since the reform and opening up, Chinese economy has become an important force in the trend of world economic integration. With the rapid development of economy, Chinese education around the world has also been warming up[1,2]. For different education groups, the traditional education model cannot meet the requirements. Online education is one of the main ways for Chinese education, because it can make it more convenient to get Chinese knowledge.

Nowadays, the existing online education model simply provided education resources to students which cannot effectively meet the practical needs of students. Only when the interests, preferences and access mode of students are better understood, it is possible to provide education resources to students according to the needs of students [3] .Student groups modeling method should be automatically constructed according to the personal needs of students. In recent years, there has been some research about student group modeling [4]. It can offer better information service only when the student groups can be effectively identified.

Many large-scale scientific computations need to use high-performance computers. However, the costs of developing and using high-performance computers are high. At the same time, specialized operating systems and programming languages are necessary for these computers, which leads to poor portability. Today, most of such researches take advantage of existing network system by performing distributed parallel computing, which achieves similar results as high-performance computing. Because of the widespread availability of network, there are many applications of distributed parallel computing in a variety of end-use situations [5-7].

Cloud computing refers to both the applications delivered as services over the Internet and the hardware and systems software in the data centers that provide those services[8]. In the core of such a non-uniform environment, facilitating interoperability, portability, and integration among heterogeneous platforms is nontrivial. Cloud computing is the integration and development of distributed computing, utility computing, virtualization technology, Web services and grid computing technology, thus it can provide the hardware services, infrastructure services, service platform, software services and storage services to various Internet application. Cloud computing can obtain the computing ability by thousands of cheap terminals via high-speed Internet, which can avoid duplication investment of large amount of hardware.

Because of simple in principle and robust, cloud computing has been applied successfully to all kinds of computation-intensive problems such as E-learning [9-11], XLearning[12], E-government[13], E-Commerce[14] and other area [15], etc.

Take full account of student's information, a new intelligent service model is proposed in this paper which can provide an efficient, consistent and personalized model for online overseas Chinese education.

The rest of this paper is organized as follows. In section 2 , the background of cloud computing is introduced. An intelligent cloud learning model is presented in section 3 . In section 4, The implementation of proposed model is introduced. Finally, some conclusions are given on the model.

\section{BRIEF INTRODUCTION TO ClOUd COMPUTING}

Cloud computing refers to both the applications delivered as services over the Internet, the hardware and systems software in the data centers that provide those services. Cloud computing has been widely recognized as the next generation's computing infrastructure. Cloud computing is a computing technology that leverages cloud's resources for "enabling ubiquitous, convenient, ondemand network access to a shared pool of configurable computing resources (e.g., networks, servers, storage, applications and services) that can be rapidly provisioned and released with minimal management effort or service provider interaction", as NIST [16] defined.

Three layer service model is a significant feature of cloud computing, including infrastructure services (IaaS), 
platform service(PaaS) and software service (SaaS). Three layer architecture of cloud computing platform is shown as figure 1 .

1) Infrastructure layer (Iaas). Users can directly access to virtual machine or other resources through the Internet and other services. IaaS provides to users not only including virtualization computing resources, but also the network bandwidth.

2) Platform layer (Paas).The platform layer can be considered the core layer of the whole cloud computing systems, including parallel programming and development environment, structured massive data, distributed storage management system and mass data distributed file system. Services provide a development platform and related services to the user, and user can directly access to related software development and running environment.

3) Application layer (SaaS). The application is refered a service available to the user. User can access service provider directly, pay on demand, which can save the cost of hardware.

\section{INTELLIGENT CLOUd LEARNING MODEL}

In this section, we proposed an intelligent learning model based on cloud computing. The intelligent cloud learning model consists cloud monitor, resources pool ,cloud server pool and students. Cloud monitor is responsible for managing resources pool and cloud server pool. Cloud nodes in server pool is responsible for the analysis of students' information, and returns the resource according to the needs of students. When computers online apply for joining the system, monitor adds it to cloud server pool. If one node is in error, monitor deletes it from the server pool. When Students submit task to the system, cloud monitor connects nodes in the server pool, sends student's information to the nodes and gathers result from the cloud nodes. If task is completed, all the threads are ceased. And all the results are summarized and exported. The intelligent cloud learning model is shown as Fig.2.

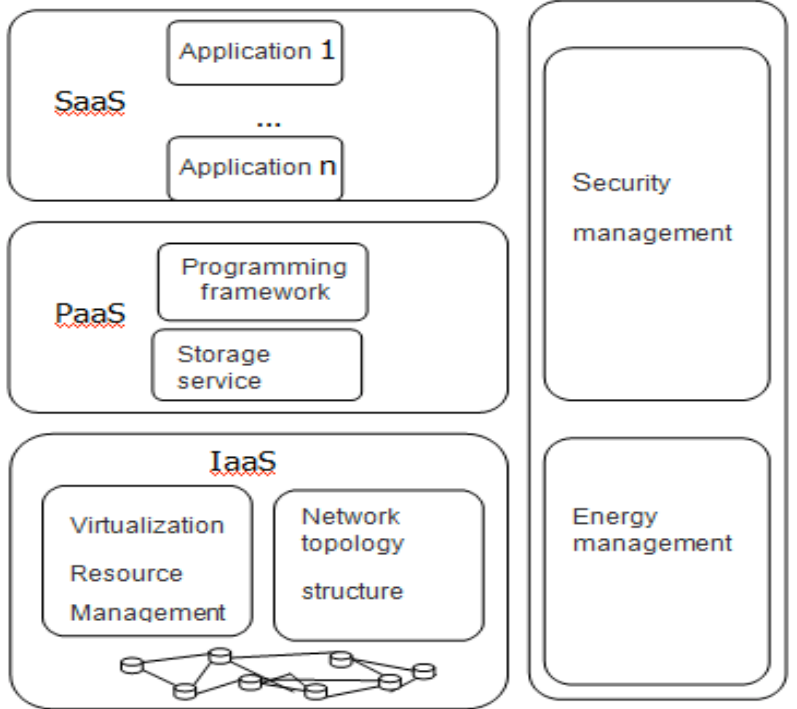

Figure 1. Three layer service model of cloud computing

The Main program with fault-tolerant Mechanism can be described as follow:

1. Cloud monitor manages server pool and adds the computers which apply for joining to pool;

2. Cloud monitor manages resource pool , and returns the resource to the students according to the needs of the student;

3. Cloud monitor runs serial task and creates a thread for each cloud node. The following steps are run in each thread;

- Nodes analysis of students' needs and report the result;

- If cloud node is in error, it puts the incomplete task blocks back to pool to let other nodes to complete.

4. If task is completed, all the threads are ceased. And all the results are summarized and exported.

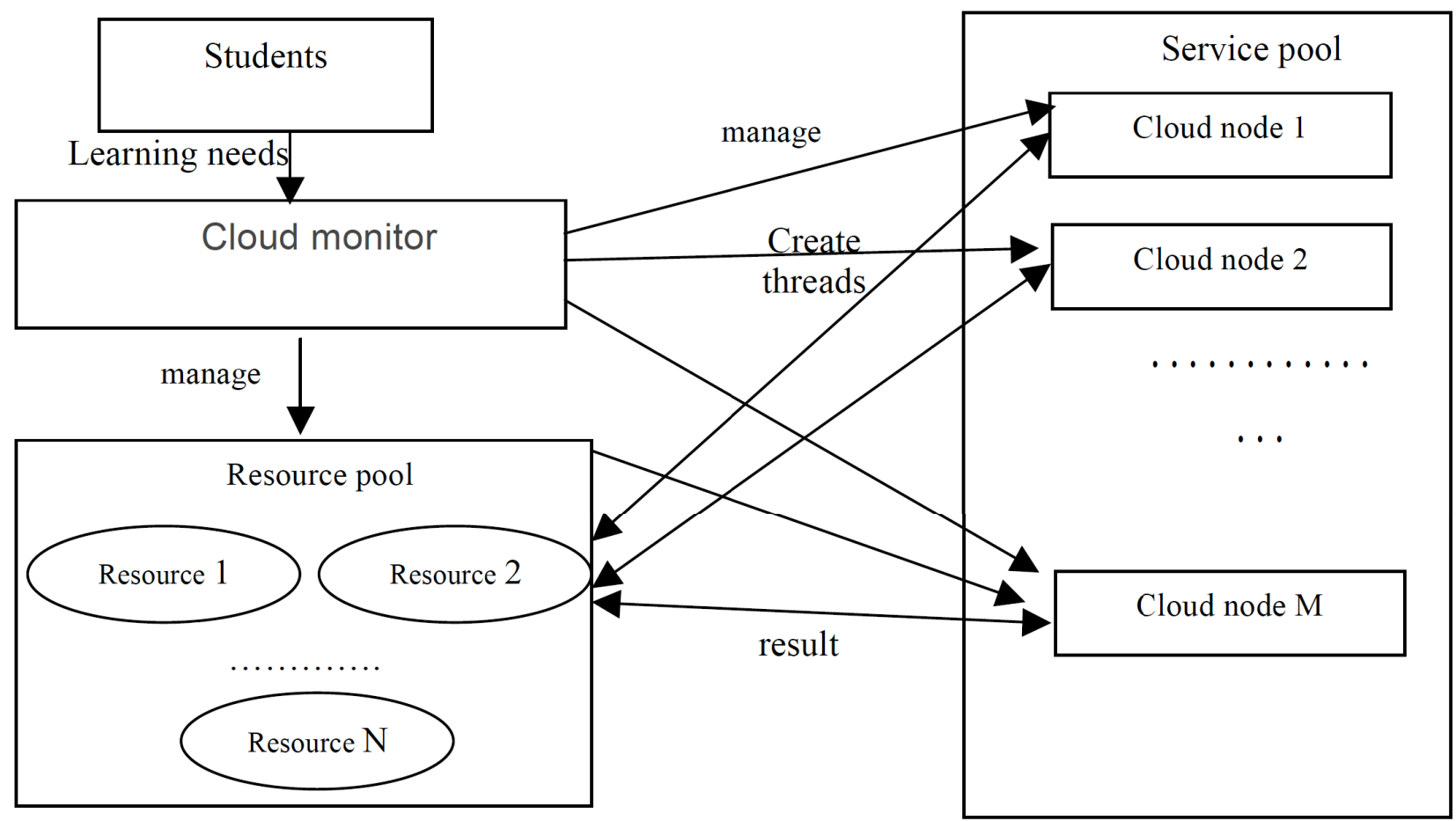

Figure 2. Intelligent Cloud Learning Mode 


\section{The Implement OF Cloud Learning Model}

In this section, the implement of proposed model is present. Firstly, we construct student information vector based on interest information, preference information and browsing history. Secondly, we cluster students into some certain groups. Finally, the implement code of cloud monitor, service pool and resource pool is given.

\section{A. Student vector space and similarity measure}

The student information can be represent as a vector $s(I, E, H)=\left(\begin{array}{ccc}I_{1} & E_{1} & H_{1} \\ I_{2} & E_{2} & H_{2} \\ \mathrm{M} & \mathrm{M} & \mathrm{M} \\ I_{n} & E_{n} & H_{n}\end{array}\right)$,

Where $I_{i}(i=1,2 \mathrm{~L} n)$ is interest information of students, $E_{i}(i=1,2 \mathrm{~L} n)$ indicates emotional and preference information of students, $H_{i}(i=1,2 \mathrm{~L} n)$ is browsing history of students. So we can better describe the relationship between student groups.

How to measure the similarity between two students is a core problem, the similarity between student $S_{i}$ and student ${ }{ }_{j}$ can be calculated by the following formula:

$$
\operatorname{similarity}\left(s_{i}, s_{j}\right)=\lambda_{1} \operatorname{sim}\left(I_{i}, I_{j}\right)+\lambda_{2} \operatorname{sim}\left(E_{i}, E_{j}\right)
$$$$
+\lambda_{i} \operatorname{sim}\left(H_{i}, H_{j}\right) \mathrm{L} \quad(1)
$$

Where $\lambda_{k}(k=1,2,3)$ are the feature weights .

$$
\begin{gathered}
\operatorname{sim}\left(I_{i}, I_{j}\right)=\sum_{k} \alpha_{k} \operatorname{sim}\left(I_{i k}, I_{j k}\right) \mathrm{L} \\
\operatorname{sim}\left(E_{i}, E_{j}\right)=\sum_{k} \beta_{k} \operatorname{sim}\left(E_{i k}, E_{j k}\right) \mathrm{L} \\
\operatorname{sim}\left(H_{i}, H_{j}\right)=\sum_{k} \gamma_{k} \operatorname{sim}\left(H_{i k}, H_{j k}\right) \mathrm{L} \\
\text { Where } \alpha_{k}=\beta_{k}=\gamma_{k}=\frac{1}{n}, \\
\operatorname{sim}\left(I_{i k}, w_{j k}\right)=\frac{\alpha}{\operatorname{dis}\left(I_{i k}, I_{j k}\right)+\alpha}, \mathrm{L} \text { (5). }
\end{gathered}
$$

Wheere $\operatorname{dis}\left(I_{i k}, I_{j k}\right)$ represents the semantic distance in the digital dictionary $\alpha$ is a constant.

$\operatorname{sim}\left(E_{i k}, E_{j k}\right)$ and $\operatorname{sim}\left(H_{i k}, H_{j k}\right)$ have similar definitions.

The realization process in cloud node is shown as Figure 3.

\section{B. Student clustering algorithm}

clustering is the task of grouping a set of objects in the same group. K-Means algorithm is one of the mostcommonly used clustering algorithms to put data into groups based on distance between nearest neighbors.

The main steps is given as follow :

1. Construct Initialization student information vector;

2. Calculate the similarity of student groups using equation (1);

3. If the similarity is within a certain threshold, we can cluster student groups on the training by using KMeans clustering;

- Choose some manner in which to initialize $m_{i}$ to be the mean of each group.

- For each example in your set, assign it to the closest group (represented by $m_{i}$ ).

- For each $m_{i}$, recalculate it based on the examples that are currently assigned to it.

- Repeat steps 2-3 until $m_{i}$ converge.

4. Output the student groups.

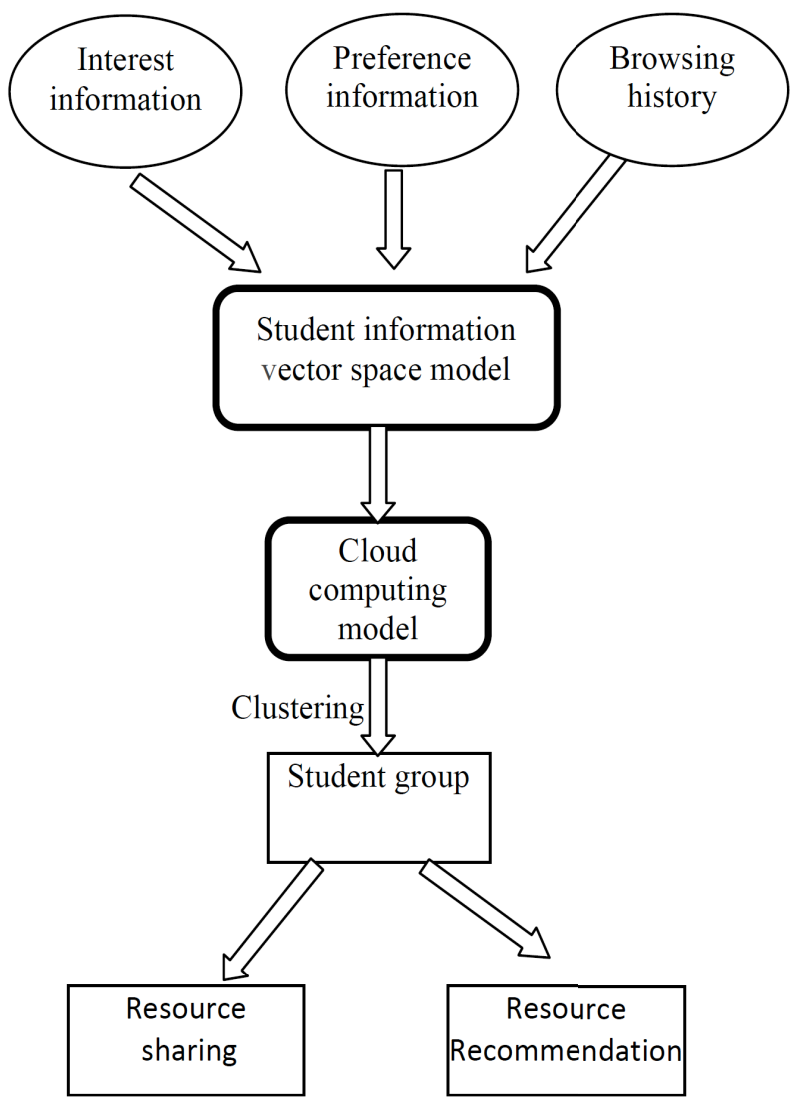

Figure 3. Analysis of students' information in cloud nodes 

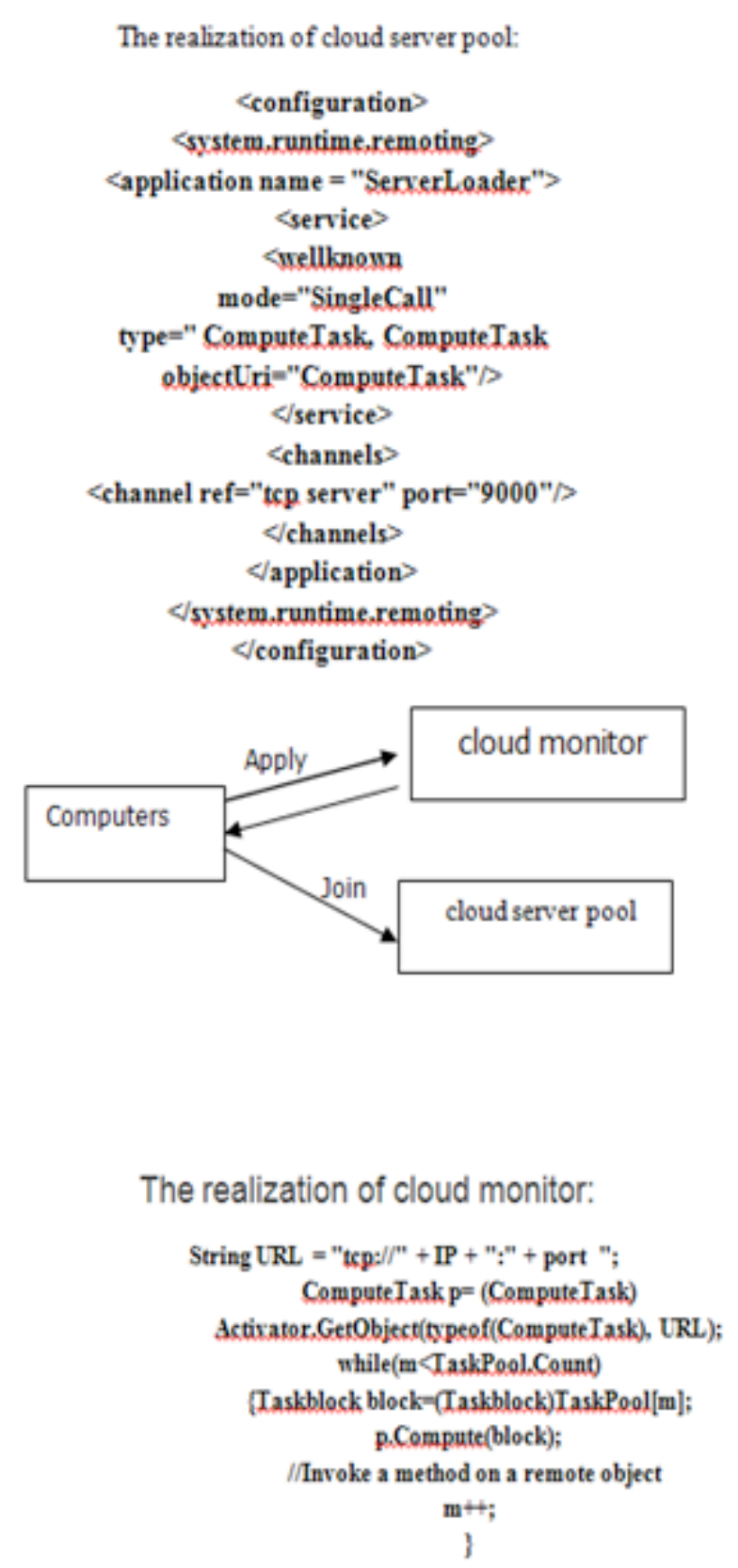

The realization of resource pool:
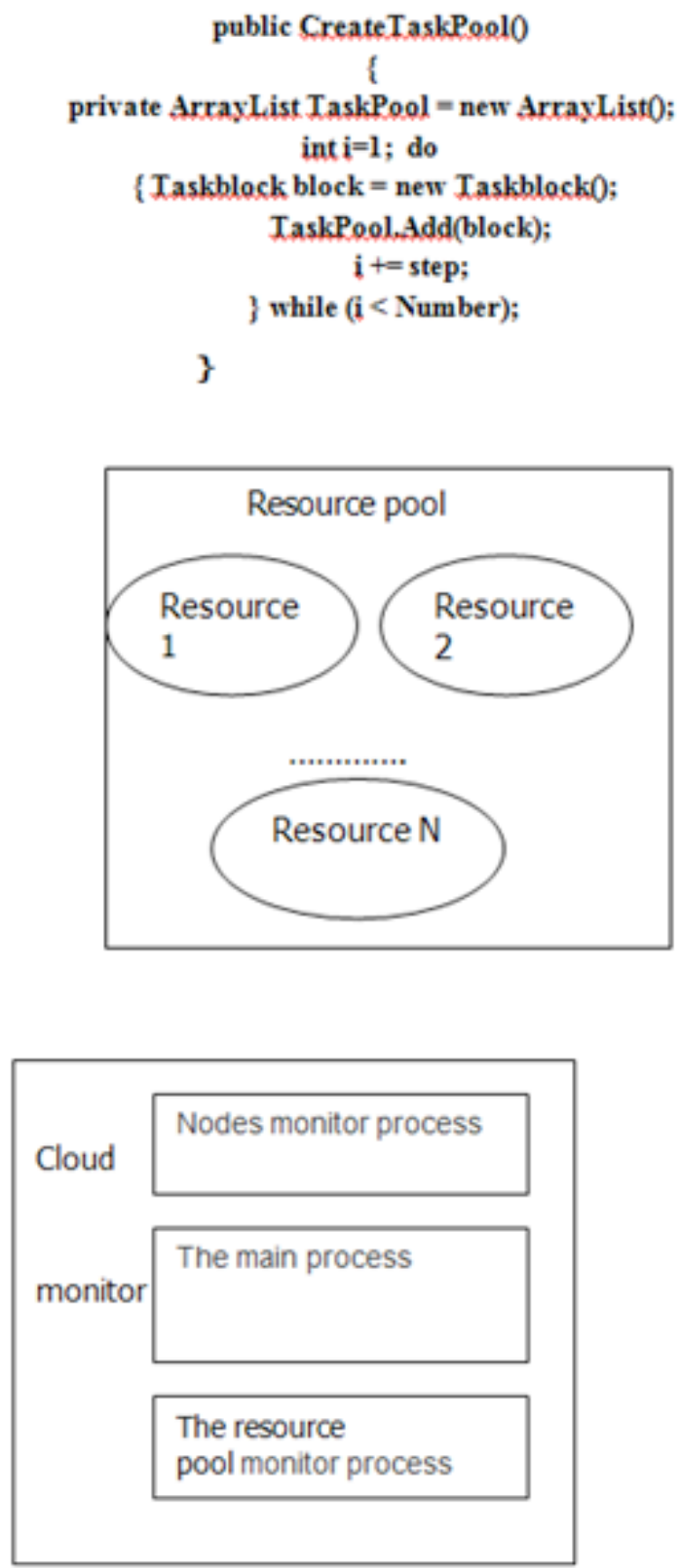

Figure 4. The realization and key code of our model

\section{The Realization And Key Code Of Our Model}

The implement code of cloud monitor, service pool and resource pool are given, as is shown in figure 4.

The cloud monitor comprises the main process, nodes monitor process and resource pool monitor process. The main process is the core of model, it can realize the main interface, the creation of multi-thread, the invocation of remote object and the summary of analysis results. The nodes monitor process is responsible for the management of cloud nodes. Any host on the network can apply to the model by registering IP address and port number. Nodes monitor process then join it to server pool. The resource pool monitor process is mainly responsible for the construction of resource pool. It can divide the resources into a number of resource block and add the resource blocks to resource pool.

\section{Some Relevant Data Stuctures}

Resource block class is a custom class. It defines the various information of resources. It contains some public member variable such as resource ID, resource basic data and resource categories. Each resource block is an instance of resource block class. Assume that the resources are composed of some resources, the cloud monitor divided the resources into a number of resource blocks. Resource ID represents the start position of resources. Resource basic data contain some resource information, it can be a structure. Resource categories describe the categories of resource. The data structure of resource class is shown as figure 5 . 


\begin{tabular}{|l|l|l|}
\hline Resource ID & Resource basic data & Resource categories \\
\hline
\end{tabular}

Figure 5. The data structure of resource class

The cloud node class is a custom class. It defines the various information of cloud node, and contains some public variable member such as IP address, port number. Each cloud node are an instance of cloud node class. The cloud monitor can determine the location of cloud node by the IP address and port number. The data structure of cloud node is shown as figure 6 .

\begin{tabular}{|c|l|}
\hline IP address & Port number \\
\hline
\end{tabular}

Figure 6. The data structure of cloud node class

\section{CONCLUSIONS}

In this paper, we present an Intelligent learning model for online overseas Chinese education based on cloud computing. We got the following conclusions.

1) The proposed model can better share the Chinese overseas resources and provide intelligent personalized information service for students.

2) The cloud service model has good extendibility. In fact, using the model to do parallel service is quite easy . For example, we can use campus network to do the experiment. One computer in the network performs as monitor and other computers can join server pool after applying to the monitor. They can download service software and join parallel service.

The learning model has a high speedup and cloud computing can utilize network resources sufficiently to implement resource sharing, and provide a good practicability and a promising future in application. In the feature, We will apply this idea to other tasks.

\section{REFERENCES}

[1] D Starr. "Chinese Language Education in Europe: the Confucius Institutes". European Journal of Education, vol 44, no 1, 2009, pp 65-82. http://dx.doi.org/10.1111/j.1465-3435.2008.01371.x

[2] Yang, R. "Soft Power and Higher Education: An Examination of China's Confucius Institutes. Globalisation", Societies and Education, vol 8, no 2, pp 235-245, 2010. http://dx.doi.org/10.1080/ 14767721003779746

[3] Vijayan Sugumaran,Veda C. "Storey,Ontologies for conceptual modeling:their creation,use, and management". Data \& Knowledge Engineering, vol.42, no.3, pp.251-271, 2002. http://dx.doi.org/10.1016/S0169-023X(02)00048-4

[4] Kerschberg,L.,Kim,W.,Scime,A, "A Semantic Taxonomy_Based Personalizable Meta-Search Agent”, Proceedings of 2th International Conference on Web Information System Engeering.Kyoto,Jappan, pp.53-62, 2001.
[5] Gusev M, Ristov S, Velkoski G, et al. "E-learning and benchmarking platform for parallel and distributed computing". International Journal of Emerging Technologies in Learning, vol.9, no.2, pp. 17-21,2014. http://dx.doi.org/10.3991/ijet.v9i2.3215

[6] Chen C W. "Neural network-based fuzzy logic parallel distributed compensation controller for structural system". Journal of Vibration and Control, vol.19, no.11, pp.1709-1727, 2013. http://dx.doi.org/10.1177/1077546312442233

[7] Brusilovsky P, Sosnovsky S, Shcherbinina O. "User modeling in a distributed e-learning architecture", User Modeling. Springer Berlin Heidelberg, pp.387-391, 2005.

[8] Ercolani G. "Cloud Computing Services Potential Analysis. An integrated model for evaluating Software as a Service". Cloud Computing, pp.77-80, 2013.

[9] Dong B, Zheng Q, Yang J, et al. "An e-learning ecosystem based on cloud computing infrastructure.Advanced Learning Technologies", Ninth IEEE International Conference on Advanced Learning Technologies, pp. 125-127,2009.

[10] Madan D, Pant A, Kumar S, et al. "E-learning based on Cloud Computing". International Journal of Advanced Research in Computer Science and Software Engineering, vol.2,no.2, 2012.

[11] Al-Zoube M, Abou El-Seoud S, Wyne M F. "Cloud computing based e-learning system". International Journal of Distance Education Technologies, vol.8, no.2, pp.58-71, 2010. http://dx.doi.org/10.4018/jdet.2010040105

[12] Xiangsheng K. "Big Data X-Learning Resources Integration and Processing in Cloud Environments". International Journal of Emerging Technologies in Learning, vol.9, no.5, pp.22-26, 2014. http://dx.doi.org/10.3991/ijet.v9i5.3783

[13] Cellary W, Strykowski S. "E-government based on cloud computing and service-oriented architecture".Proceedings of the 3rd international conference on Theory and practice of electronic governance. ACM, pp.5-10,2009.

[14] Yang X, Pan T, Shen J. "On 3G mobile e-commerce platform based on cloud computing". 3rd IEEE International Conference on. Ubi-media Computing, pp.198-201,2010.

[15] Li L, Zhang M. "The strategy of mining association rule based on cloud computing". 2011 International Conference on Business Computing and Global Informatization. pp.475-478, 2011. http://dx.doi.org/10.1109/BCGIn.2011.125

[16] P. Mell and T. Grance, "The NIST definition of cloud computing". 2011. [Online]. Available: http://csrc.nist.gov/publications/nist pubs/800-145/SP800-145.pdf

\section{AUTHORS}

Zhehuang Huang is a lecturer in the School of Mathematics Sciences, Huaqiao University. His research interest covers E-learning, intelligent algorithm, machine learning and data fusion.

Xiaodong Shi: Corresponding author. He is a Professor in Cognitive Science Department, Xiamen University. His research interest covers machine translation and machine learning.

This work was supported by the National Natural Science Foundation of China (Grant No. 61005052), the Fundamental Research Funds for the Central Universities (Grant No. 2010121068) and the science and technology project of Quanzhou (Grant No.2012Z91). Submitted 28 November 2014. Published as resubmitted by the authors 21 February 2015. 